\title{
The organisation of spatial and temporal relations in memory
}

\section{Renante Rondina II, Kaitlin Curtiss, Jed A. Meltzer, Morgan D. Barense \& Jennifer D. Ryan}

To cite this article: Renante Rondina II, Kaitlin Curtiss, Jed A. Meltzer, Morgan D. Barense \& Jennifer D. Ryan (2016): The organisation of spatial and temporal relations in memory, Memory, DOI: $10.1080 / 09658211.2016 .1182553$

To link to this article: http://dx.doi.org/10.1080/09658211.2016.1182553

册 Published online: 16 May 2016.

Submit your article to this journal $\pi$

Q View related articles ¿

View Crossmark data $₫$ 


\title{
The organisation of spatial and temporal relations in memory
}

\author{
Renante Rondina $\|^{a, b}$, Kaitlin Curtissa ${ }^{a}$, Jed A. Meltzer ${ }^{a, b}$, Morgan D. Barense ${ }^{b}$ and Jennifer D. Ryan ${ }^{a, b, c}$

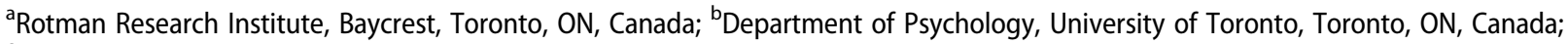 \\ 'Department of Psychiatry, University of Toronto, Toronto, ON, Canada
}

\begin{abstract}
Episodic memories are comprised of details of "where" and "when"; spatial and temporal relations, respectively. However, evidence from behavioural, neuropsychological, and neuroimaging studies has provided mixed interpretations about how memories for spatial and temporal relations are organised-they may be hierarchical, fully interactive, or independent. In the current study, we examined the interaction of memory for spatial and temporal relations. Using explicit reports and eye-tracking, we assessed younger and older adults' memory for spatial and temporal relations of objects that were presented singly across time in unique spatial locations. Explicit change detection of spatial relations was affected by a change in temporal relations, but explicit change detection of temporal relations was not affected by a change in spatial relations. Younger and older adults showed eye movement evidence of incidental memory for temporal relations, but only younger adults showed eye movement evidence of incidental memory for spatial relations. Together, these findings point towards a hierarchical organisation of relational memory. The implications of these findings are discussed in the context of the neural mechanisms that may support such a hierarchical organisation of memory.
\end{abstract}

\section{ARTICLE HISTORY}

Received 13 July 2015

Accepted 20 April 2016

\section{KEYWORDS}

Relational memory; spatial and temporal relations; eye movements; ageing;

short-term memory
Episodic memories are punctuated with details regarding the "where" and "when" of personal events. These spatial and temporal relations, respectively, tend to co-vary in daily life: events occurring in a certain place may be closely connected in time, and events that occur close in time may also occur closely in space. However, an open question remains with respect to the organisation of temporal and spatial relations in memory. Memory may be organised in a hierarchical manner such that the memory for one type of relation directly impacts memory for relations in the other dimension in a unidirectional manner (cf. Healy, 1975), memory for spatial and temporal relations may be fully interactive, or information regarding spatial and temporal relations may be maintained independently within memory.

Prior research on the effect of different encoding instructions on subsequent memory may speak to the organisation of relations in memory. In a series of immediate cued-recall studies (Delogu, Nijboer, \& Postma, 2012a, 2012b), temporal order recall was impaired when participants were instructed to encode spatial and temporal relations compared to when participants were instructed to encode temporal relations only. By contrast, spatial recall was unaffected when there was the additional requirement to encode temporal information. Additionally, temporal order recall was substantially greater than spatial recall under single-dimension encoding instructions, whereas temporal order recall was similar to that of spatial recall in the dual-encoding conditions. Together, these findings point towards a hierarchical organisation of memory with temporal information primarily encoded, as there was no cost to spatial memory when there was an additional requirement to encode temporal information, whereas there was a cost to temporal memory when there was an additional requirement to encode spatial information. Furthermore, memory performance for spatial information under single encoding conditions was equivalent to memory performance for temporal information under dual-encoding conditions, which suggests that temporal information may be "easier" to maintain, whether that ease of maintenance is due to a strategic shift in the nature of encoding, or due to the organisation of spatial and temporal information in memory.

Other behavioural findings in the literature suggest that spatial and temporal information may be maintained separately in memory. For instance, in a speeded classification task, spatial recall was not influenced by the temporal position of the probe item at study, and temporal recall was not influenced by its spatial location (Dutta \& Nairne, 1993); thus, memory for one type of relation 
was not influenced by memory for the other type of relation, suggesting that information regarding spatial and temporal relations is maintained separately in memory. Similar conclusions were drawn by Van Asselen, Van der Lubbe, and Postma (2006), who demonstrated worse recall in an immediate cued-recall task when participants were instructed to encode one relation and were subsequently tested on the other, as compared to performance for the intentionally encoded relation. Worse recall under incidental versus intentional encoding conditions may indicate that information may not be obligatorily encoded, and further, is maintained separately in memory. However, when participants were required to maintain both spatial and temporal information, changes in spatial position affected temporal recall, and changes in temporal order affected spatial recall (Dutta \& Nairne, 1993). Taken together, these results suggest that the encoding instructions may determine the extent to which information regarding spatial and temporal relations is maintained in memory, and/or the extent to which information regarding one set of relations impacts the other-at least as indexed by the response times and accuracy of explicit reports (Dutta \& Nairne, 1993).

Neuroimaging studies have revealed the unique neural regions that support spatial and temporal memory. Spatial memory has been associated with neural responses in the medial temporal lobes (see Spiers \& Maguire, 2007, for a review of neuroimaging evidence; see Kessels, de Haan, Kappelle, \& Postma, 2001, for a review of lesion studies in humans), whereas temporal memory has been associated with neural responses in the frontal lobes (Amiez \& Petrides, 2007; Kessels et al., 2001). These findings are consistent with neuropsychological studies that have dissociated patterns of spared versus impaired spatial and temporal memory: individuals with frontal lesions have been shown to have larger impairments on a temporal context memory task compared to individuals with temporal lobe lesions, whereas those with temporal lobe lesions, but not frontal lesions, showed impairments on spatial context memory (Kopelman, Stanhope, \& Kingsley, 1997). Such findings may support a proposal that spatial and temporal information rely on separate memory traces. However, there are neural responses in the hippocampus that occur during spatial and temporal relational memory tasks (Kumaran \& Maguire, 2006) and amnesic individuals who have lesions to the hippocampus have impaired spatial as well as temporal relational memory (Konkel, Warren, Duff, Tranel, \& Cohen, 2008). Additionally, place cells (O'Keefe \& Nadel, 1978), which fire when an animal occupies a particular location in an environment, as well as time cells (Eichenbaum, 2014), which fire at successive moments in temporally structured experiences, have both been detected in the hippocampus. Thus, neuroimaging and neuropsychological findings suggest that spatial and temporal information may rely on partially overlapping memory traces that are mediated by the hippocampus.
In summary, the literature on the organisation of temporal and spatial relations in memory is mixed, with both evidence of interaction and evidence of independence of relations in explicit reports, and evidence for at least a partially overlapping neural trace for spatial and temporal relations from neuroimaging and neuropsychological studies. It may be the case that there is some amount of inter-dependence of relations in memory, as suggested by the neuroimaging and neuropsychological work, but that inter-dependence may not always be readily available for conscious introspection. Previous studies have relied solely on direct, explicit reports to probe memory for spatial and temporal relations (Delogu et al., 2012a, 2012b; Kahana, Howard, Zaromb, \& Wingfield, 2002; Kopelman et al., 1997; Olson et al., 2004; Sekuler, McLaughlin, Kahana, Wingfield, \& Yotsumoto, 2006; Van Asselen et al., 2006). However, it is possible that interactions between spatial and temporal memory may be present in indirect measures of memory, even when they are absent from direct measures of memory. That is, memory for incidentally encoded relations may be present, and may influence the memory for the intentionally tested relation, but may not necessarily be available for, or impact, conscious expression. Therefore, in the present study, we examined indirect as well as direct indices of memory (cf. Ryan, Althoff, Whitlow, \& Cohen, 2000; Ryan, Hannula, \& Cohen, 2007) to determine the extent to which spatial and temporal relations may be obligatory encoded and interact with each other.

A short delay memory paradigm was adapted from our previous work (Olsen, Rondina, Riggs, Meltzer, \& Ryan, 2013; Ryan \& Villate, 2009) to examine the encoding and subsequent retrieval of spatial and temporal relations in younger and older adults. Older adults were included in the present study as to the best of our knowledge, there have been no studies that have compared the interactions in memory between spatial and temporal relations in older adults. Moreover, given that deficits in spatial and temporal relational memory are typically observed with increasing delays for older adults (see Old \& Naveh-Benjamin, 2008, for a review; but see Parkin, Walter, \& Hunkin, 1995), likely due to declining function in the hippocampus, comparing differences in performance between younger and older adults may reveal the extent to which there is a hierarchical organisation of relations in memory.

Measures derived from eye movement monitoring were used to indirectly examine the online binding of spatial and temporal relations during the study phase. During the test phase, explicit reports were used as a direct index of memory, and measures derived from eye movement monitoring were used as indirect indices of memory for spatial and temporal relations (Pathman \& Ghetti, 2014, 2015; Ryan et al., 2000; Ryan, Leung, TurkBrowne, \& Hasher, 2007; Ryan \& Villate, 2009). The inclusion of eye movement monitoring in the present paradigm allowed us to examine differential encoding behaviour depending on the instructions to encode spatial versus 
temporal relations, and to examine the influence of spatial or temporal relations online during retrieval, even when such information may not ultimately reflected in the final output of retrieval processing, as indexed by explicit reports. Participants were presented with a set of objects, presented singly across time (Figure 1). Following a delay the objects were again presented, but the objects could be arranged with either a change to their relative spatial relations or a change in their temporal order of presentation, or both. Participants were asked to detect either a change in spatial or temporal relations, but, critically, one or both types of manipulations could be present. In this way, we could examine whether relational memory for one dimension would impact either direct or indirect expression of relational memory in the other dimension, thereby revealing the potentially hierarchical organisation of information in memory.

To the extent that relational information exists in a hierarchical fashion in memory, there should be a one-way dependence, such that a change in one type of relation affects the ability to detect a change in the other, but not vice versa. Additional evidence for a possible hierarchical organisation of relational information in memory can be obtained by examining performance in older adults, who typically experience declines in relational memory. Ageing may differentially affect memory for one type of relation compared to the other, which would support a hierarchical account of memory for temporal and spatial relations, whereas a similar effect of ageing on both types of relations, in the absence of any evidence for a one-way dependence of memory, would suggest a non-hierarchical organisation. Examination of memory in a population known for declining relational memory function, in concert with findings from younger adults, would collectively provide insight into the potential hierarchical organisation of memory in the brain.

\section{Experiment 1}

\section{Methods}

\section{Participants}

Sixty-four healthy adults from the Toronto community with normal neurological histories and normal or corrected-tonormal vision participated in the study. Sixteen younger adults (age: $M=21.8$ years, $\mathrm{SD}=2.4$, education: $M=15.2$ years, $\mathrm{SD}=1.5$ ) and 16 older adults (age: $M=70.4$ years, $\mathrm{SD}=4.0$; education: $M=16$ years, $\mathrm{SD}=2.0$ ) were assigned to the Spatial Group in which they were instructed to remember, and were subsequently tested on, the relative spatial relations among the presented objects. Sixteen younger adults $(M=22.4$ years, $S D=2.9$; education: $M=16.3$ years, $S D=2.3$ ) and 16 older adults (age: 70.2 years, $S D=5.7$; education: $M=17.3$ years, $S D=4.2$ ) were assigned to the Temporal Group in which they were instructed to remember, and were subsequently tested on, the temporal order of the presented objects. Univariate analyses revealed no difference in age between instruction groups, and no difference in education between age or instruction groups (all $p s>.07$ ).

\section{Stimuli and design}

Stimuli consisted of 600 coloured novel objects and 200 patterned backgrounds $(1024 \times 768$ pixels $)$ and were among a set used in prior work (Olsen et al., 2013; Ryan \& Villate, 2009). The novel objects were created using Corel Draw v.12 and were designed so as to have minimal resemblance to real-world objects, in order to discourage use of associated verbal labels. Stimuli were grouped into trial-unique sets containing three objects and a patterned background. Each trial contained a study phase and a test phase. During the study phase, each of the three images was presented singly on the background in locations unique for the trial (see Figure 1(a)). A given object location could repeat across the sets of stimuli, but the combination of locations of all three objects was never repeated across sets. During the test phase the same three objects were presented on the same background as seen during the study phase, but all objects were presented in new absolute spatial locations (see Figure 1(b-e)). All test trials involved a change in the objects' absolute positions to prevent participants from relying on local luminance changes to detect changes in spatial position. In addition to a change in absolute position, one of the objects could also change its relative spatial position with respect to the other objects (spatial manipulation). Spatial manipulations occurred equally often in each of the ordinal positions across all manipulated spatial trials The test displays could also contain a manipulation in temporal order, such that either two or three objects were changed in their ordinal position (temporal manipulation); all combinations of ordinal manipulations were equally represented across the manipulated temporal trials.

Specifically, for $25 \%$ of trials (50 trials), the three objects were presented during the test phase with the same relative spatial relations and in the same temporal order as had been presented at study (intact spatial/intact temporal trials; see Figure $1(b)$ ). For another $25 \%$ of trials, the relative spatial relations among the objects were disrupted from the study to the test phase, whereas the temporal order of object presentation remained unchanged (manipulated spatial/intact temporal trials; see Figure 1(c)). For another $25 \%$ of trials, the three objects were presented during the test phase with the same relative spatial relations, but with a different temporal order, than what had been presented at the study phase (intact spatial/manipulated temporal trials; see Figure $1(d)$ ). For a final set of $25 \%$ of trials, both the relative spatial relations and the temporal order of the presented objects were disrupted from the study to the test phase (manipulated spatial/manipulated temporal trials; see Figure 1(e)). Across all images, object positions were distributed across the whole screen, and all stimulus sets were counterbalanced across participants such that each was seen equally often as each of the different test trial types, and 


\section{Study Phase}

(a)

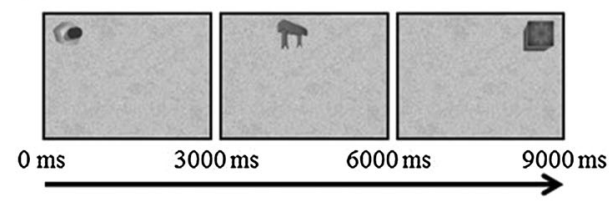

Test Phase

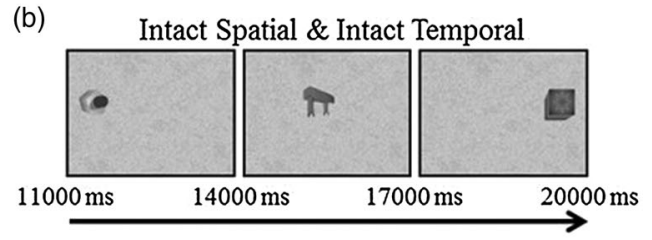

(c) Manipulated Spatial \& Intact Temporal

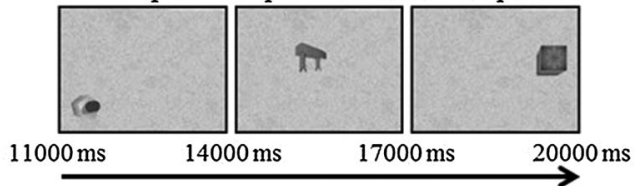

(d) Intact Spatial \& Manipulated Temporal

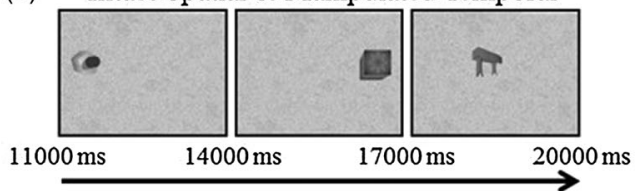

(e) Manipulated Spatial \& Manipulated Temporal

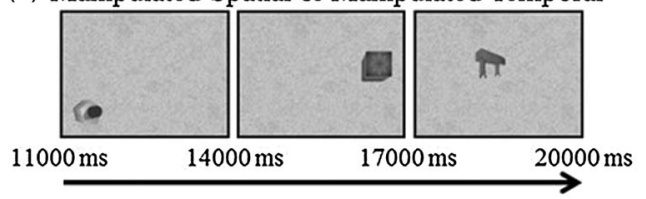

Figure 1. Example of stimuli and procedures. (a) Study Phase. Participants were presented with three objects overlaid on a patterned background. Objects appeared one at a time in different locations on the screen followed by a visual mask. Participants were asked to remember either the spatial relations or temporal relations among the objects. (b-e) Test Phase. Participants were presented with three objects which appeared one at a time, as in the study phase, but now in new absolute spatial locations. In addition, trials could also contain a spatial and/or temporal manipulation to the objects from what had been presented in the study phase: (b) intact spatial and intact temporal relations (c) intact spatial and manipulated temporal relations (d) manipulated spatial and intact temporal relations (e) manipulated spatial and manipulated temporal relations. Numbers above the arrows indicate the onset of each image. Spatial Group was asked "Was there a change to the relative position?" Temporal Group was asked "Was there a change to the order?".

manipulated versions of the sets for one group of participants became the original sets for another (and vice versa).

\section{Procedure}

There were 200 trials in total divided into four viewing blocks of 50 trials each, with the four trial types (intact spatial/intact temporal; intact spatial/manipulated temporal; manipulated spatial/intact temporal; and manipulated spatial/manipulated temporal) presented in a random order. Each trial was initiated by a central fixation screen. During the study phase, the three study images were presented singly for $3000 \mathrm{~ms}$ each, followed by a visual mask presented for $2000 \mathrm{~ms}$. Participants in the Spatial Group were told to remember the relative spatial relations among the studied objects, whereas participants in the Temporal Group were told to remember the temporal relations among the studied objects. In the test phase, the objects were again presented singly for 3000 ms each. A question screen was presented immediately following the test presentation of the three objects, which asked "Was there a change to the relative position?" (Spatial Group) or "Was there a change to the order?" (Temporal Group), and remained on the screen until the participant made a "yes" or "no" response with their index fingers.

\section{Behavioural data analysis}

Accuracy. The following metrics were calculated to measure the accuracy to detect a manipulation in the tested relation: adjusted accuracy (proportion of hits minus the proportion false alarms), and $A^{\prime}$. For the Spatial Group, hits were defined as manipulated spatial trials that were correctly identified as "manipulated", and false alarms were defined as intact spatial trials that were incorrectly identified as "manipulated". For the Temporal Group, hits were defined as manipulated temporal trials that were correctly identified as "manipulated", and false alarms were defined as intact temporal trials that were incorrectly identified as "manipulated". A' measures were also calculated as they have been argued to be less biased measures of population $d^{\prime}$ for hit rates and false alarm rates of 1 and 0 (Donaldson, 1993; Mueller \& Weidemann, 2008; Mueller \& Zhang, 2005; Stanislaw \& Todorov, 1999; Weidemann \& Mueller, 2008; Zhang \& Mueller, 2005).

To determine whether a change in the untested relation (i.e. intact versus manipulated temporal relations for the Spatial Group, and intact versus manipulated spatial relations for the Temporal Group) would affect memory for the directly tested relation, a three-way mixed-design ANOVA was conducted on each measure of accuracy with age (younger adults, older adults) and instruction 
group (spatial, temporal) as between-subject factors, and the untested trial type (intact, manipulated) as a withinsubject factor.

\section{Eye movement data acquisition and analysis}

Acquisition. Eye movements were measured with a SR Research Ltd. Eyelink II head-mounted eyetracker which recorded eye movements at a rate of $500 \mathrm{~Hz}$ and with a spatial resolution of 0.1 degrees. A 9-point calibration was performed at the start of each block followed by a 9point calibration accuracy test. Calibration was repeated if the error at any point is more than 1 degree. Drift

(a) Study Phase: "Looking Back"

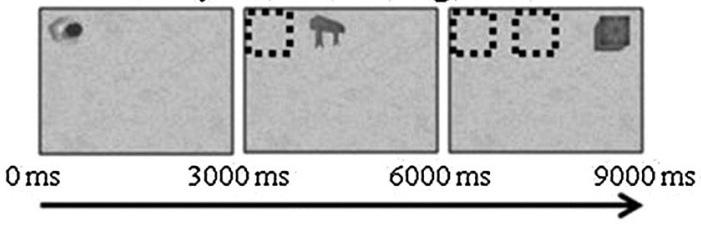

(b) Test Phase: "Looking Back"

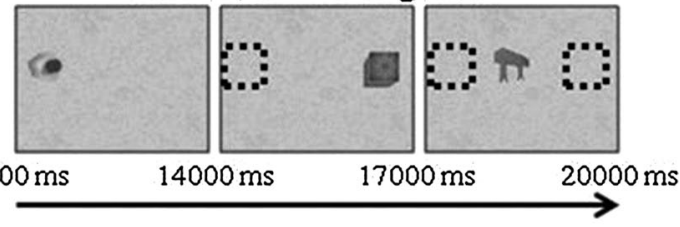

(c) Test Phase: Critical Spatial Region - Filled

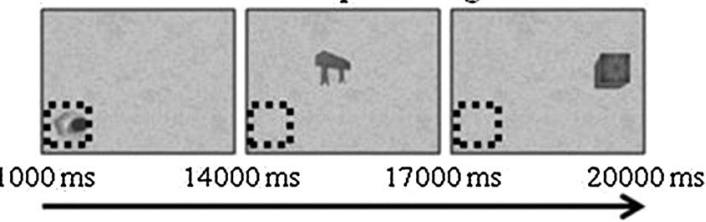

(d) Test Phase: Critical Spatial Region - Empty

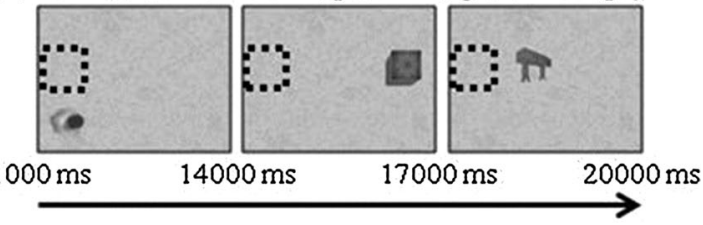

Figure 2. Example of regions of interest for different analyses. (a) Study Phase: "Looking Back". To examine online binding of spatial and/or temporal relations during the study phase, viewing towards the previously filled regions, as indicated by the dashed lines, was summed across the trial. (b) Test Phase: "Looking Back". Example of an intact spatial/manipulated temporal trial is shown. To examine the extent to which temporal manipulations may affect online viewing behaviour during the test phase, cumulative viewing to the previously filled regions, as indicated by the dashed lines, was examined. It was predicted that a temporal manipulation may increase "looking back" behaviour in an effort to compare the presented temporal order with what had been maintained in memory. (c-d) Example of a manipulated spatial trial in which the first study object has been moved relative to the other two objects. To examine memory for the previously studied spatial relations, we assessed viewing to the location where the critical object (i.e. the object that had undergone a change in its relations) is currently presented (c. Test Phase: Critical Spatial Region-Filled), and to the location where the critical object would have been had the relative spatial positions remain unchanged from study to test (d. Test Phase: Critical Spatial Region-Empty). corrections were performed at the beginning of each trial when necessary.

"Looking back" behaviour. Our prior work (Ryan \& Villate, 2009) has shown that, across the study phase, participants tend to direct their viewing back to regions that had been previously occupied by objects, which we interpreted as reflecting the ongoing binding of relations among the study objects. To investigate online binding behaviour during the study phase, regions of interest were created that outlined the regions surrounding the previously presented objects. The proportion of total viewing time that was directed to previously filled regions across the study phase ("looking back") was calculated (see Figure 2(a)). To determine whether there were any differences in online binding as a result of age or instruction group, a two-way ANOVA was conducted on "looking back" behaviour during the study phase with age and instruction as between-subject factors.

For the images presented during the test phase, regions of interest were also created that outlined the regions surrounding the previously presented objects, and "looking back" behaviour was calculated (see Figure 2(b)). If "looking back" behaviour reflects online binding, it was expected that there would be an increase in this behaviour during manipulated (either spatial or temporal) compared to intact trials, as participants would likely compare the presented relations with those stored in memory, and perhaps update the representation with the new relations. Thus, a four-way mixed-design ANOVA was conducted on the proportion of viewing to the previously filled regions during the test phase with age and instruction as betweensubject factors, and spatial trial type (intact, manipulated) and temporal trial type (intact, manipulated) as withinsubject factors.

Viewing to critical spatial regions. Our prior work (Ryan et al., 2000; Ryan \& Villate, 2009) has shown that participants directed greater viewing to an object that has undergone a change in its relative spatial relations, and its corresponding empty region (i.e. where the object used to be) during manipulated trials compared to intact trials, suggesting that participants were comparing the externally presented relations with what had been stored in memory. To similarly assess memory for spatial relations in the test phase of the present experiment, regions of interest were also created that outlined the regions surrounding the object that had undergone a spatial manipulation (filled region; see Figure $2(\mathrm{c})$ ), and the empty region that would have been occupied had the spatial relations remained the same (empty region; see Figure 2(d)). Spatial manipulations occurred equally often at each ordinal position, thus viewing to the critical filled and empty regions was examined across the entire test phase. Given that our previous work has shown an age-related impairment in viewing to regions that had undergone a manipulation in spatial relations (Ryan, Leung, et al., 2007), we expected to find 
an interaction between age and spatial trial type. Thus, a three-way mixed-design ANOVA was conducted on the proportion of viewing to the critical spatial filled and empty regions, separately, with age and instruction group as between-subject factors, and spatial trial type as a within-subject factor.

\section{Threshold levels}

Five separate ANOVAs were conducted in this study; in order to set the experiment-wise error to $a=0.05$, all behavioural and eye movement data were analysed at a Bonferonni-corrected criterion level of $p<.05 / 5=.01$ for significant effects, and a Bonferonni-corrected criterion level of $p<.10 / 5=.02$ for marginal effects.

\section{Results}

\section{Accuracy}

To determine whether a change in one relation affected memory for the other relation, a three-way mixed-design ANOVA was conducted on each measure of accuracy with age and instruction as between-subject factors, and untested trial type as a within-subject factor (Figure 3). The Temporal Group (adjusted accuracy: $M=0.92, \mathrm{SD}=$ 0.09; $A^{\prime}: M=0.98 S D=0.03$ ) was more accurate than the Spatial Group (adjusted accuracy: $M=0.64, S D=0.18 ; A^{\prime}$ $=0.88, \mathrm{SD}=0.07)$, adjusted accuracy: $F_{(1,60)}=70.13, p$ $<.001, \eta_{p}^{2}=0.54 ; A^{\prime}: F_{(1,60)}=52.93, p<.001, \eta_{p}^{2}=0.47$. There was no main effect of age group (all $p s>.02$ ) and no interaction with age group (all $p s>.04$ ).

Participants had greater accuracy for the trials for which the untested relations were intact (intact untested trials; adjusted accuracy: $M=0.80, S D=0.19 ; A^{\prime}: M=0.94, S D=$ $0.06)$ than for manipulated untested trials (adjusted accuracy: $\left.M=0.76, \mathrm{SD}=0.22 ; \mathrm{A}^{\prime}: M=0.92, \mathrm{SD}=0.08\right)$, adjusted accuracy: $F_{(1,60)}=7.81, p<.01, \eta_{p}^{2}=0.12 ; A^{\prime}: F_{(1,60)}=9.13$, $p<.01, \eta_{p}^{2}=0.13$. However, this effect was modulated by marginal and significant interactions between untested trial type and instruction group (adjusted accuracy: $F_{(1,60)}$ $=6.23, \quad p=.015, \quad \eta_{p}^{2}=0.09 ; \quad A^{\prime}: \quad F_{(1,60)}=9.13, \quad p<.01$, $\left.\eta_{\mathrm{p}}^{2}=0.13\right)$, which revealed a simple effect of untested trial type for the Spatial Group (adjusted accuracy: $t_{(31)}=$ 2.77, $\left.p<.01, d=0.49 ; A^{\prime}: t_{(31)}=3.00, p<.01, d=0.53\right)$ who had greater accuracy on intact temporal trials (adjusted accuracy: $M=0.68, \mathrm{SD}=0.18 ; \mathrm{A}^{\prime}: M=0.90, \mathrm{SD}=0.07$ ) compared to manipulated temporal trials (adjusted accuracy: $M=0.60, \mathrm{SD}=0.21 ; \mathrm{A}^{\prime}: M=0.87, \mathrm{SD}=0.09$ ), but no simple effect of untested trial type for the Temporal Group (all $t s<1)$. Thus, a change in temporal relations affected memory for spatial relations, but a change in spatial relations did not affect the memory for temporal relations. This suggests a one-way dependence in memory: memory for temporal relations may be independent of spatial information, but memory for spatial relations may inherently include, or require, temporal information. This finding points towards a hierarchical organisation in memory in which temporal relations can affect spatial relations, and that such organisation is preserved with ageing.

Due to concerns of a potential ceiling effect, we sought to determine whether the reported pattern of results was consistent across different levels of performance. We compared the highest performing tertile of participants in all groups to the lowest performing tertile of participants in all groups. Critically, there were no two-way interactions between tertile and untested trial type, and no three-way interactions between tertile, instruction, and untested trial type (all $p s>.08$ ). Therefore, the interaction between instruction and untested trial type was consistent across all levels of performance.

\section{"Looking back": study phase}

To examine online binding during the study phase (Ryan \& Villate, 2009), a two-way ANOVA was conducted on the proportion of viewing to the previously filled regions across the study phase (Figure 4(a)) with age and instruction group as between-subject factors (Figure 4(b)). The Spatial Group directed a greater amount of viewing to the previously filled regions $(M=0.12, S D=0.06)$ compared to the Temporal Group $\left(M=0.07, \mathrm{SD}=0.01 ; F_{(1,60)}=28.57\right.$, $\left.p<.001, \eta_{p}^{2}=0.32\right)$. There was no significant main effect of age group and no interaction with age group (all $p s>.15)$. These results suggest that younger and older adults alike may have engaged in more effortful online binding of spatial than of temporal relations, or that the encoding of temporal relations may rely on different cognitive strategies that do not necessarily require the encoding of spatial position, such as relying on verbal coding of temporal order (e.g. first, second, and third).

\section{"Looking back": test phase}

To examine the influence of either spatial or temporal relational manipulations on online binding, a four-way mixeddesign ANOVA was conducted on the proportion of viewing to the previously filled regions during the test phase (Figure 5(a)) with age and instruction group as between-subject factors, and spatial trial type and temporal trial type as within-subject factors (Figure 5(b)). The Spatial Group $(M=0.08, S D=0.02)$ engaged in more "looking back" behaviour than the Temporal Group $(M=0.06$, $\left.\mathrm{SD}=0.01 ; F_{(1,60)}=14.50, p<.001, \eta_{p}^{2}=0.20\right)$. There was no significant main effect of spatial trial type, and no significant interaction with spatial trial type (all $p s>.06$ ). Participants in both groups directed greater viewing to the previously filled regions on manipulated temporal trials $(M=0.08, \mathrm{SD}=0.02)$ than on intact temporal trials $(M=$ $0.06, \mathrm{SD}=0.02$; main effect of temporal trial type: $F_{(1,60)}=$ 52.35, $\left.p<.001, \eta_{p}^{2}=0.47\right)$. There was no significant interaction between temporal trial type and instruction group $(F<1)$, no significant main effect of age group $(F<1)$, and no significant interaction with age group (all $p s$ $>$.09). These results suggest a change in temporal relations affected online binding behaviour, regardless of task 


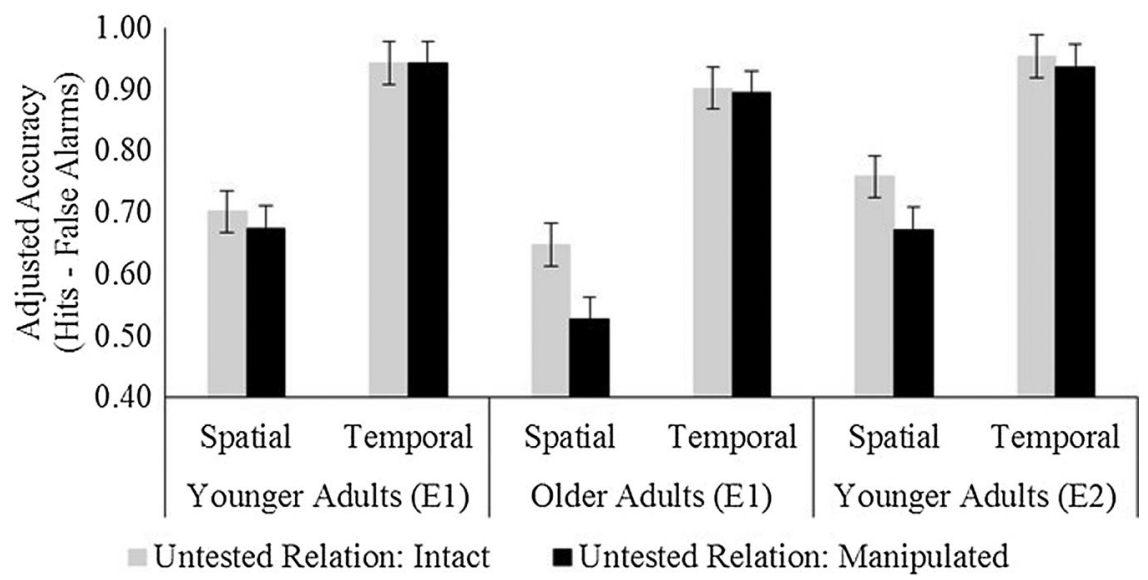

Figure 3. Adjusted accuracy (hits minus false alarms) to detect a manipulation in the tested relation (i.e. space for the Spatial Group; temporal order for the Temporal Group), separated by whether the relations in the untested dimension were manipulated (i.e. temporal order for the Spatial Group; space for the Temporal Group). Younger and older adults in Experiment 1 (E1) and younger adults in Experiment 2 (E2). In both experiments, participants in the Spatial Group were significantly worse at identifying a spatial manipulation if the trial also contained a temporal manipulation. Accuracy to detect a manipulation to temporal order for participants in the Temporal Group was not affected by the presence of a spatial manipulation. Error bars represent standard errors.

instructions, and that incidental memory for temporal relations was preserved in older adults.

\section{Viewing to the critical spatial region: filled}

A preliminary four-way ANOVA including temporal trial type as a within-subject factor showed no significant main effect of temporal trial type, and no significant interactions between spatial and temporal trial types (all $p$ s $>$.12). Thus, temporal trial type was omitted from the following analyses. To examine memory for previously viewed spatial relations, a three-way mixed-design ANOVA was conducted on the proportion of viewing to the critical filled region (Figure 6(a)), for which an object had undergone a change in its relative spatial relations, with age and instruction as between-subject factors, and spatial trial type as a within-subject factor (Figure 6(b)). The Temporal Group directed greater viewing to the filled region $(M=0.25, S D=0.03)$ than the Spatial Group $\left(M=0.23, \quad \mathrm{SD}=0.04 ; \quad F_{(1,60)}=8.83, \quad p<.01, \quad \eta_{\mathrm{p}}^{2}=0.13\right)$. Younger adults directed greater viewing to the filled region $(M=0.25, \mathrm{SD}=0.03)$ than the older adults $(M=0.23, \mathrm{SD}=$ $\left.0.04 ; F_{(1,60)}=9.02, p<.01, \eta_{p}^{2}=0.13\right)$. There was no main effect of spatial trial type $(p>.22)$. An interaction between spatial trial type and age group $\left(F_{(1,60)}=6.40, p\right.$ $\left.=.01, \eta_{\mathrm{p}}^{2}=0.10\right)$ revealed that younger adults directed more viewing to the filled region on manipulated $(M=$ $0.26, \mathrm{SD}=0.03)$ versus intact spatial trials $(M=0.25, \mathrm{SD}=$ $0.03), t_{(31)}=3.47, p<.01, d=0.61$. In contrast, this effect was not present in older adults (spatial trial type: $t<1$ ), despite the fact that older adults showed above chance explicit memory for detection of spatial changes. There were no other interactions with spatial trial type (all $F_{s}<$ 1). These results demonstrate the likely obligatory effect of memory on eye movement behaviour, as younger adults showed memory for spatial relations regardless of the task they were given. Whereas younger and older adults revealed similar direct expressions of memory for spatial relations in their explicit reports, only younger adults exhibited eye movement behaviours that were indicative of an indirect expression of memory for spatial relations. This pattern of findings suggest that older adults' memory for spatial relations may not be as robust or obligatory as that of younger adults.

\section{Viewing to the critical spatial region: empty}

The same analyses as the above were conducted with the critical empty region (Figure 7(a)), which would have contained an object had the spatial relations been maintained from study to the test (results shown in Figure 7(b)). As observed in the filled region analyses, the Spatial Group directed more viewing to the empty region $(M=0.02$, SD $=0.01)$ than the Temporal Group $(M=0.01, S D=0.01$; $\left.F_{(1,60)}=29.69, p<.001, \eta_{p}^{2}=0.33\right)$. In contrast to what we observed for the filled region, older adults directed more viewing to the empty region $(M=0.02, S D=0.01)$ than the younger adults $\left(M=0.01, \mathrm{SD}=0.01 ; F_{(1,60)}=9.54, p<.01\right.$, $\left.\eta_{p}^{2}=0.14\right)$. There was no significant interaction between age and instruction group $(p>.02)$. A significant main effect of spatial trial type $\left(F_{(1,60)}=33.89, p<.001\right.$, $\left.\eta_{\mathrm{p}}^{2}=0.36\right)$, revealed greater viewing of the empty region on manipulated $(M=0.02, S D=0.02)$ compared to intact spatial trials $(M=0.01, S D=0.01)$. This effect was driven by the Spatial Group, as revealed by a significant interaction between spatial trial type and instruction group $\left(F_{(1,60)}=29.93, p<.001, \eta_{p}^{2}=0.33\right)$, and a simple effect of spatial trial type for the Spatial Group $\left(t_{(31)}=6.08\right.$, $p<.001, d=1.07)$ that was not present for the Temporal Group $(t<1)$. There were no other significant interactions with spatial trial type (all $p s>.07$ ). These results demonstrate that both younger and older adults directed greater viewing to the critical empty region when it had undergone a manipulation, but this effect was observed only under intentional encoding instructions. 
(a) Study Phase: "Looking Back"
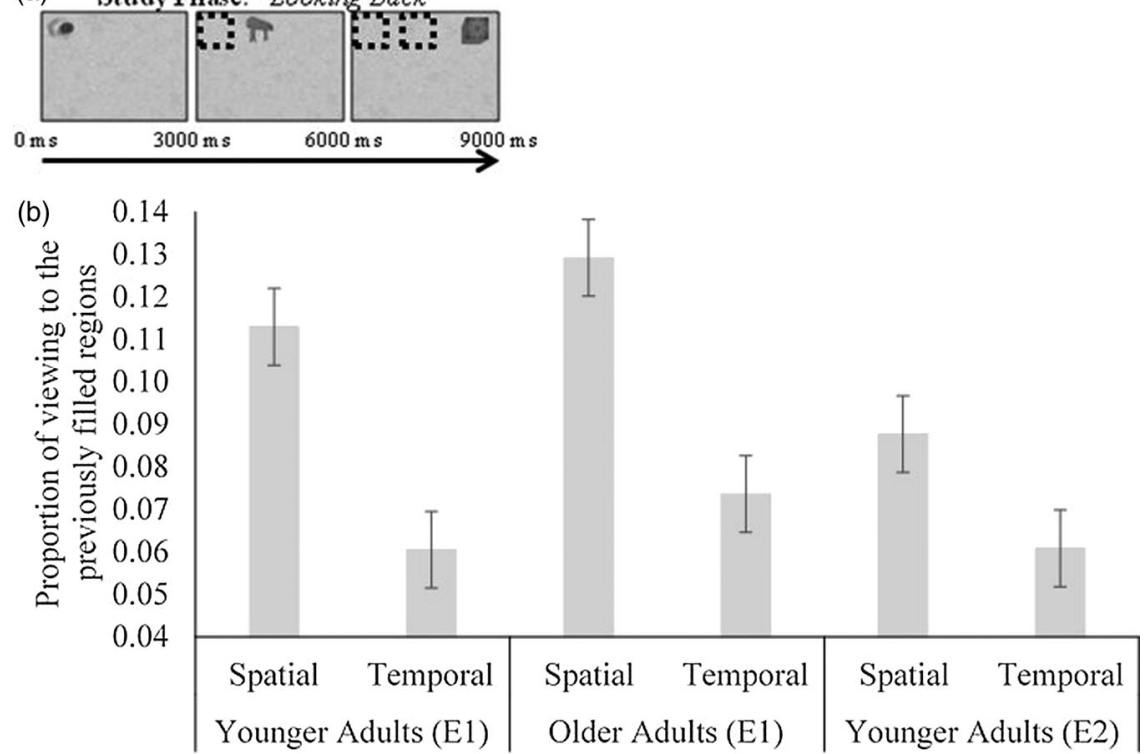

Figure 4. Proportion of viewing to the previously filled regions during the study phase ("looking back" behaviour). (a) Example study phase is shown. Dashed lines indicate previously filled regions. (b) Younger and older adults in Experiment 1 (E1) and younger adults in Experiment 2 (E2). In both experiments, participants in the Spatial Group directed greater viewing to the previously filled regions than did participants in the Temporal Group, indicating more effortful online binding, or the use of different encoding strategies. Error bars represent standard errors.

The present experiment revealed that memory for spatial information was affected by manipulations that were made to temporal information. Participants were less accurate to detect a spatial manipulation when there was a concomitant change in temporal relations, however, the ability to detect a change in temporal relations was not affected by changes in spatial information. Moreover, older adults showed intact temporal memory, but impaired spatial memory, relative to younger adults, as indicated by eye movements. Together, these results suggest a one-way dependence regarding the relations in memory with temporal relations being primary, providing an anchor for spatial relations. However, in the present design, absolute spatial positions were changed from the study to test phase, but the absolute temporal pacing was not. Thus, it is possible that memory for
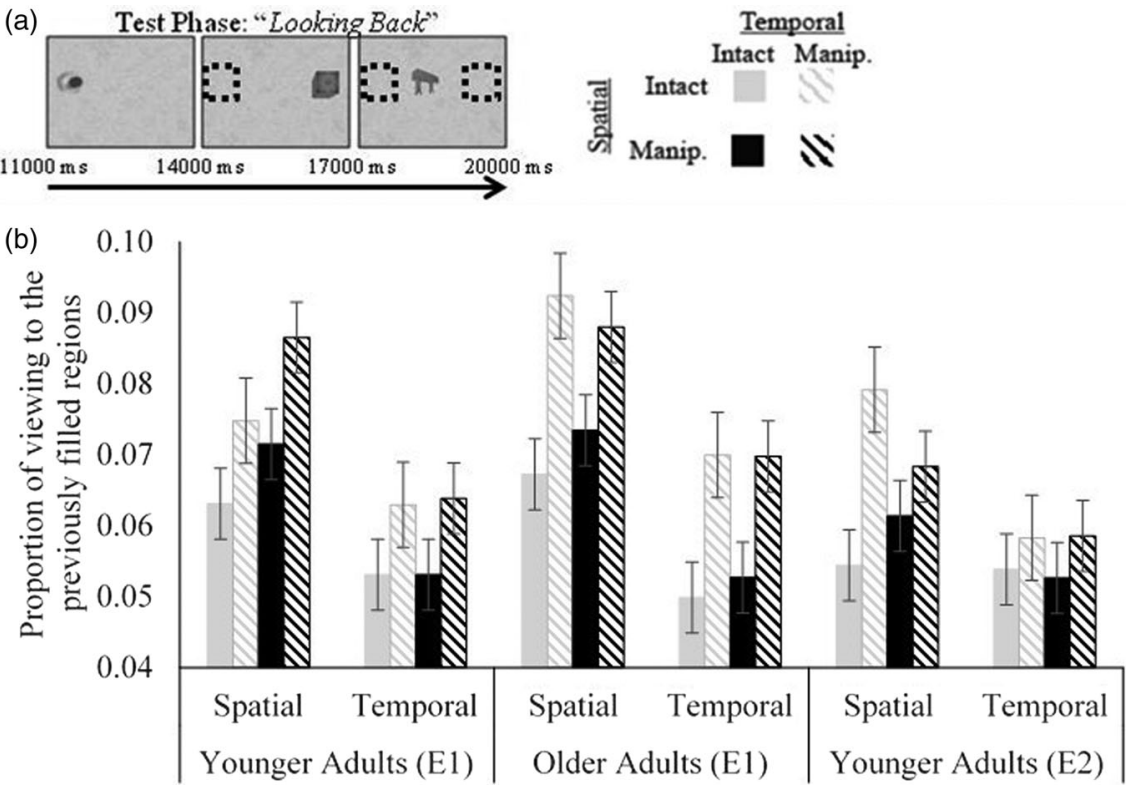

Figure 5. Proportion of viewing to the previously filled regions during the test phase ("looking back" behaviour). (a) Example of an intact spatial/manipulated temporal trial is shown. Dashed lines indicate previously filled regions. (b) Younger and older adults in Experiment 1 (E1) and younger adults in Experiment 2 (E1). In all groups, participants directed greater viewing to the previously filled regions for manipulated compared to intact temporal trials, regardless of age or instruction group. Error bars represent standard errors. 
(a) Test Phase: Critical Spatial Region-Filled
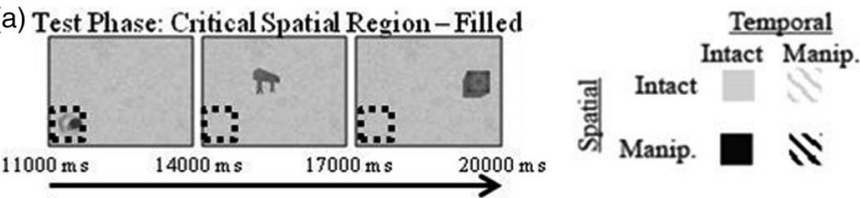

(b)

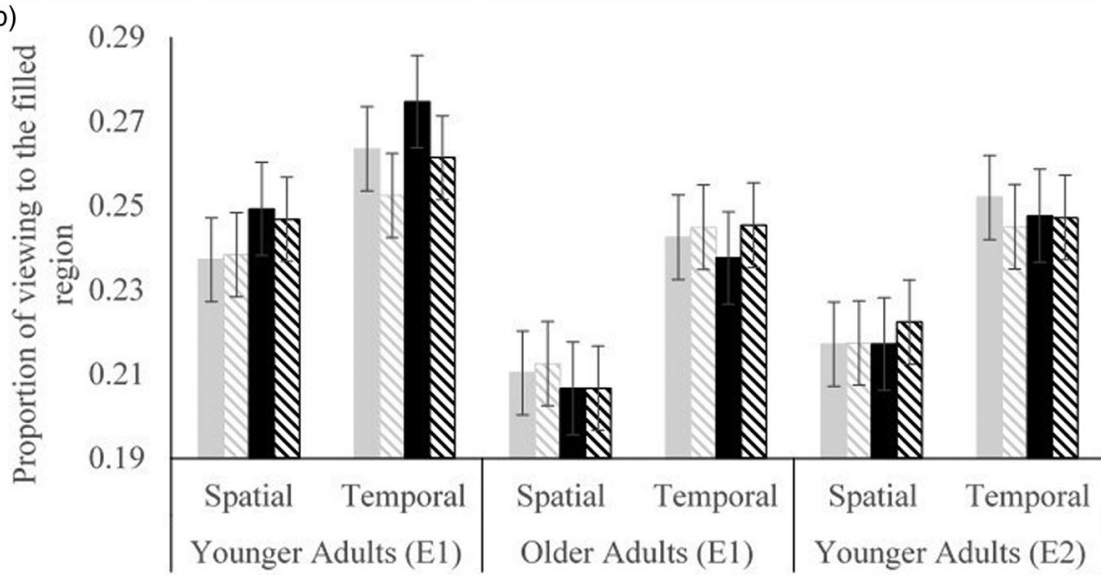

Figure 6. Proportion of viewing to the filled critical spatial region. (a) Example of a manipulated spatial trial in which the first study object has been moved relative to the other two objects. Dashed lines indicate the location of the critical object that has undergone a change in its spatial relations. (b) Younger and older adults in Experiment 1 (E1) and younger adults in Experiment 2 (E2). Younger adults in E1 directed greater viewing to the filled region for manipulated compared to intact spatial trials regardless of instruction group. Older adults in E1 and younger adults in E2 did not show this effect of spatial memory. Error bars represent standard errors.

temporal relations would have been affected by changes in spatial relations if the absolute temporal pacing were disrupted. Alternatively, if temporal information provides an anchor for spatial relations, then spatial memory should be affected by a change in the temporal pacing. A second experiment was conducted to explore this possibility.

\section{Experiment 2}

A second experiment was conducted to further test the hypothesis that temporal information provides a foundation for spatial relations, as well as to address the limitations of the first experiment. Particularly, although the absolute spatial position of the objects changed from study to test, there was no corresponding change in temporal pacing. The constant temporal pacing from study to test may have allowed participants to use the temporal order as a way to organise the trial structure and the observed spatial information. To control for this potential confound, the present experiment tested younger adults on an adaptation of the previous experiment in which the temporal pacing was altered during the test phase.

\section{Methods}

\section{Participants}

Sixteen younger adults (age: $M=24.7$ years, $\mathrm{SD}=4.9$, education: $M=16.0$ years, $S D=2.0)$ were assigned to the Spatial Group, and 16 younger adults $(M=22.6$ years, $\mathrm{SD}=$ 3.6; education: $M=15.8$ years, $S D=2.2$ ) were assigned to the Temporal Group in which they were instructed to remember, and were subsequently tested on, the temporal order of the presented objects. Univariate analyses revealed no difference in age between instruction groups, and no difference in education between age or instruction groups (all $p s>.17$ ).

\section{Stimuli, design, and procedures}

The same stimuli and task designs as Experiment 1 were used. The order of events in the structure of a trial were also the same as in Experiment 1 (study phase, two second delay, test phase, and response screen), except that the temporal pacing was varied from study to test. During the study phase, each object was presented for $3 \mathrm{~s}$ each. However, during the test phase, each object was presented for a variable duration of 2.5 to $3.5 \mathrm{~s}$, with no two objects having the same duration within the same trial, while keeping the total duration of each test trial fixed at $9 \mathrm{~s}$. Participants were given the same instructions as in Experiment 1.

\section{Results}

Findings regarding looking back behaviour at test (see Figure 5) and viewing to the critical filled region (see Figure 6) are reported below. Findings regarding accuracy (Figure 3), looking back behaviour during the study phase (Figure 4), and viewing to the critical empty region (Figure 7) are presented in the figures, but are not reported in detail for the sake of brevity as there were no significant main effects or interactions with experiment version (all ps $>.03$ ) and the pattern of findings did not differ from what was observed in Experiment 1. 
(a) Test Phase: Critical Spatial Region-Empty
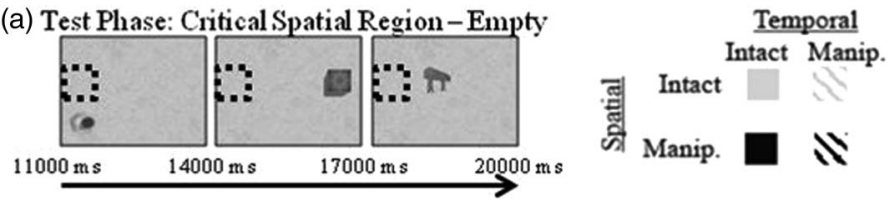

(b)

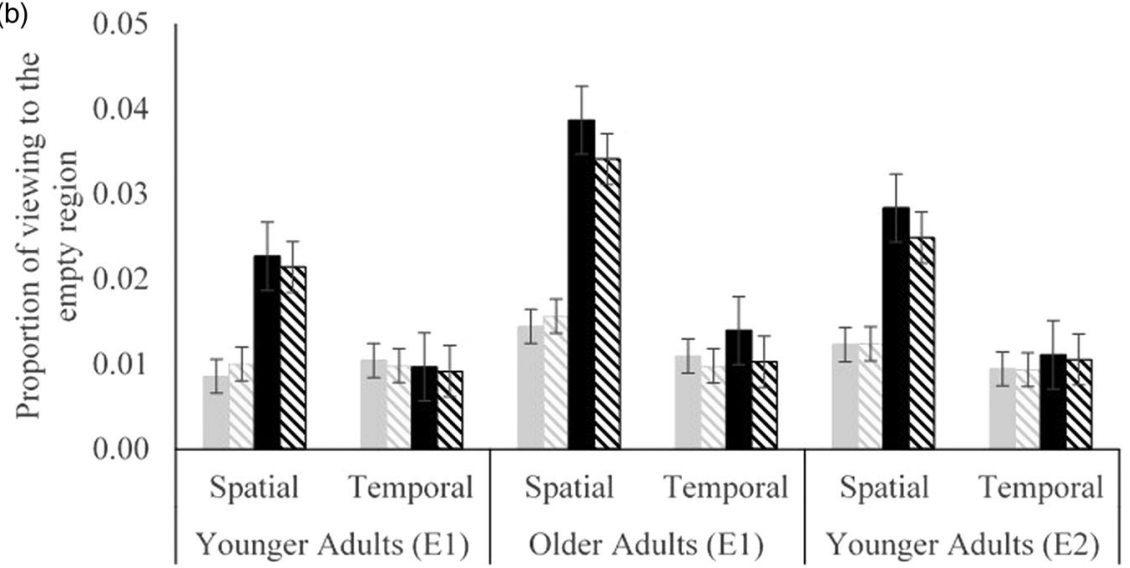

Figure 7. Proportion of viewing to the empty critical spatial region. (a) Example of a manipulated spatial trial in which the first study object has been moved relative to the other two objects. Dashed lines indicate the location where the critical object would have been had the relative spatial positions remained unchanged from study to test. (b) Younger and older adults in Experiment 1 (E1) and younger adults in Experiment 2 (E2). In both experiments, participants in the Spatial Group directed greater viewing to the empty region for manipulated compared to intact spatial trials, regardless of age group. Participants in the Temporal Group did not show this effect of spatial memory. Error bars represent standard errors.

\section{"Looking back": test phase}

Looking back behaviour during the test phase was submitted to a four-way ANOVA with task instruction and experiment version as between-subject factors, and spatial and temporal trial type as within-subject factors (see Figure 5). Participants showed more looking back behaviour for manipulated temporal trials $(M=0.06, S D=$ $0.01)$ than intact temporal trials $(M=0.07, S D=0.02)$, $F_{(1,60)}=29.90, p<.01, \eta_{p}^{2}=0.33$. There were no interactions with temporal trial type (all $p \mathrm{~s}>.03$ ). However, there was a three-way interaction between task instruction, experiment version, and spatial trial type, $F_{(1,60)}=7.05$, $p=.01, \eta_{\mathrm{p}}^{2}=0.11$, such that the younger adults in the spatial group of Experiment 1 showed more looking back behaviour on manipulated spatial trials $(M=0.08, S D=$ $0.02)$ than on intact spatial trials $(M=0.07, S D=0.01)$, $t_{(15)}=3.63, p<.01$, but there was no such effect of spatial trial type for any of the other groups (all $p s>.30$ ). This suggests that a disruption in the temporal pacing disrupted eye movement evidence of memory for spatial changes. Participants may use temporal order, and absolute pacing, as an anchor to organise the trial structure, such that there is a hierarchical organisation of spatial and temporal relations in memory.

\section{Viewing to the critical spatial region: filled}

The proportion of viewing to the critical filled region was submitted to a three-way ANOVA with task instruction and experiment version as between-subject factors, and spatial trial type as a within-subject factor (see Figure 6). There was no main effect of spatial trial type $\left(F_{(1,60)}=\right.$ 4.94, $p=.03, \eta_{p}^{2}=0.08$ ), and no interaction between experiment version and spatial trial type $\left(F_{(1,60)}=3.76, p\right.$ $>.05, \eta_{p}^{2}=0.06$ ). However, as Experiment 1 showed a simple effect of spatial trial type for younger adults, these results suggest that a disruption in temporal pacing disrupted eye movement evidence of memory for spatial changes. Together with the results from the looking back behaviour during the test phase in Experiment 2, this suggests that participants may use temporal order, and absolute pacing, as an anchor to organise the trial structure, such that there is a hierarchical organisation of spatial and temporal relations in memory.

\section{Discussion}

In the present study, we used eye movement monitoring and explicit reports to examine age-related changes to the encoding and retrieval of intentional and incidental memory for spatial and temporal relations, and to understand any interdependencies between spatial and temporal information in memory. Participants who were instructed to remember the spatial relations among the objects engaged in more "looking back" behaviour than participants who were instructed to encode the temporal relations, suggesting that there is a difference in the demand of online binding resources to form memories of spatial versus temporal relations. By asking participants to detect a manipulation in one dimension and varying whether a manipulation occurred in the other dimension, we found that a change in temporal relations affected memory for spatial relations, but not vice versa, as indexed by explicit reports. This effect of temporal relations on memory for spatial relations was maintained even when 
the absolute temporal pacing was disrupted from the study to test phases. Also, there was a differential effect of ageing on memory such that eye movements revealed a significant spatial memory impairment in older adults relative to younger adults. In contrast, older adults' memory for temporal order was similar to that of younger adults as indexed by both eye movements and explicit reports. All together, these findings point towards a hierarchical organisation of memory, in which temporal relations can affect spatial memory, and that spatial memory disproportionately declines with ageing.

Consistent with long-standing findings in eye movement research (Yarbus, 1967), different encoding instructions resulted in different viewing patterns during the study phase. As the presentation of the objects unfolded, participants who were instructed to remember the relative spatial positions of the objects tended to look back towards the locations that had been previously occupied; however, instructions to remember the temporal order of the objects lessened this effect. Previously, we have suggested that this "looking back" behaviour serves to bind the relations among the objects (Ryan \& Villate, 2009). Differences in "looking back" as a function of task instructions suggests that the encoding of spatial relations may require more effortful online processing than the encoding of temporal order, or that encoding of temporal relations may rely more on a verbal, rather than a spatial, code (Gmeindl, Walsh, \& Courtney, 2011; Healy, 1975). Interestingly, no age-related differences were observed in "looking back", suggesting that older and younger adults in this task employed similar encoding strategies (Blachstein, Greenstein, \& Vakil, 2012; Kahana et al., 2002; Olson et al., 2004; Sekuler et al., 2006).

Despite different encoding instructions and its resultant differences in "looking back" during the study phase, participants in all groups, regardless of task instruction or age, showed similar memory for temporal relations, as evidenced by explicit reports and eye movement behaviour. In particular, participants directed increased viewing to the locations of the previously presented objects when a change had occurred in the temporal relations from the study to the test phase. This suggests that participants were comparing the currently presented temporal relations to the original relations that had been stored in memory and subsequently retrieved. This evidence for temporal memory retrieval and comparison occurred even under incidental conditions, suggesting the encoding and subsequent use of temporal relations may be obligatory, and that there is sparing of temporal relational memory with ageing. The sparing of temporal relational memory with ageing is consistent with previous studies that have found intact memory for temporal order in ageing over a short delay (Blachstein et al., 2012; Kahana et al., 2002; Sekuler et al., 2006).

By contrast, ageing was associated with changes in memory for spatial relations, as indexed by eye movements. Whereas younger adults directed increased eye movements towards an object (the filled region; see Figure 6) if it had undergone a change in its spatial relations, regardless of task instructions, this effect was not observed in older adults. However, younger and older adults alike directed increased eye movements towards the empty region (see Figure 7 ) that would have contained an object had it not undergone a change in its relations, but in both groups this effect occurred only under intentional instructions to remember the spatial relations. Such findings of increased viewing to regions of spatial manipulations are consistent with previous work (Hollingworth, Williams, \& Henderson, 2001; Parker, 1978; Ryan et al., 2000; Ryan \& Cohen, 2004; Ryan \& Villate, 2009), and suggest that the original spatial relations had been retrieved from memory and were being compared to the relations that were externally presented (Ryan \& Cohen, 2004). It may be the case that viewing of the empty region is more dependent on ongoing task demands, and is more useful when performance relies on spatial processing. However, the current findings suggest that viewing of the object itself that had been changed in its spatial relations may reflect obligatory spatial processing and memory, which is not influenced by temporal information, given that the younger adults showed eye movement-based memory effects of spatial relations regardless of task demands. Ageing, by contrast, appears to be associated with a decline in the expression of spatial relational memory through eye movements, consistent with our previous work (Ryan, Leung, et al., 2007). This suggests that older adults require greater effort, a more deliberate, or a fundamentally different encoding strategy, to successfully encode spatial relations, whereas younger adults may succeed with either less effort, or an obligatory encoding process.

Findings from the explicit reports suggest that there may be a one-way dependence in relational memory. Specifically, participants who were instructed to detect a change in the relative spatial relations were less accurate if a change had also occurred in the presented temporal relations. This suggests that temporal relations are obligatorily encoded, subsequently retrieved, and may be used to support memory for spatial relations. In contrast, the ability to detect a change in temporal relations was not affected by a concurrent change in spatial relations, even in Experiment 2 when the absolute temporal pacing was disrupted. Together with the findings of a dissociation between spatial and temporal relational memory as measured by eye movement behaviours, these findings provide converging evidence for a hierarchical structure of memory.

Temporal relations may be able to affect spatial memory due to the nature of encoding itself. For example, in order to encode a static image containing numerous objects placed at considerable distances from each other, attention must be shifted, either covertly or overtly, through the use of multiple eye fixations that inspect each object with foveal vision. Consequently, relations can be bound in memory as each object is inspected in turn (cf. Ryan \& Villate, 2009). Similarly, an event or an episode unfolds 
over time, thereby requiring that objects and/or locations are encoded relative to a temporal order as changes occur (i.e. cause and effect). Thus, the contents of memory are necessarily embedded within a temporal order, and the present study provides novel evidence that this temporal information is used to support memory for the spatial relations among objects. Consistent with this notion, previous studies have shown that information regarding the temporal order of fixations in a scan path may be retained in memory and used to subsequently support recognition itself (Brandt \& Stark, 1997; Laeng \& Teodorescu, 2002; Walker-Smith, Gale, \& Findlay, 1977).

A hierarchical organisation of memory is supported by robust findings of incidental memory for temporal order in free recall studies (see Kahana, Howard, \& Polyn, 2008). Item list recall tends to be organised such that items that were studied close together in time are subsequently recalled close together in time at test, typically in the same order. These effects are thought to be driven by a slowly changing temporal context that is associated with each item (Howard \& Kahana, 2002). When an item is retrieved from memory, its associated context is retrieved as well, which then activates other items associated with similar contexts. These effects of temporal contiguity also predict episodic memory performance (Sederberg, Miller, Howard, \& Kahana, 2010).

The notion of a privileged role for temporal relations in the organisation of memory is consistent with recent ideas regarding the functional role of neural oscillations. Specifically, the temporal order of events can be encoded in the timing of their activations relative to the phase of low-frequency (i.e. theta) oscillations (cf. Lisman \& Buzsáki, 2008; Lisman \& Idiart, 1995; Lisman \& Redish, 2011; O'Keefe \& Recce, 1993). Theta oscillations could be a neural mechanism that provides a temporal context onto which items may be associated (Howard \& Kahana, 2002). Indeed, recent studies have shown that theta power increases during the delay period of a working memory task were associated with temporal order memory performance (Hsieh, Ekstrom, \& Ranganath, 2011; Roberts, Hsieh, \& Ranganath, 2013). Theta oscillations may also enable the binding of relations between objects by providing a neural "ambience" onto which higher frequency oscillations can co-activate specific item and location representations (Fell \& Axmacher, 2011; Jensen \& Colgin, 2007; Sauseng, Griesmayr, Freunberger, \& Klimesch, 2010). Indeed, incremental theta power increases that occurred across an encoding phase have also predicted accuracy on a short delay spatial memory task, similar to the present study (Olsen et al., 2013). These theta power changes at encoding may reflect the use of temporal order to support the encoding of spatial relations. With respect to the current study, theta oscillations may be a neural mechanism by which temporal order affects the memory for spatial relations, and produces the one-way dependence in which a change in temporal order affected the memory for spatial relations.
The notion of a hierarchical structure in memory also extends previous studies regarding the role of the hippocampus in relational memory. Although traditionally thought to be specifically involved in memory over long delays, recent findings suggest that the hippocampus is also involved in the memory for relations over short delays (Olsen, Moses, Riggs, \& Ryan, 2012). The hippocampus is also a major source of theta oscillations (Axmacher, Cohen, et al., 2010; Axmacher, Henseler, et al., 2010; Kahana, Sekuler, Caplan, Kirschen, \& Madsen, 1999; Lega, Jacobs, \& Kahana, 2012; Raghavachari et al., 2001), and hippocampal theta power increases predicted performance in a short delay spatial memory task (Olsen et al., 2013). The hippocampus is also known to undergo structural and/or functional changes in ageing (Driscoll et al., 2003; Erickson et al., 2010; Rondina et al., 2015), which is consistent with our findings of impaired spatial memory in older adults. In contrast, a dorsal fronto-parietal network has been implicated in memory for temporal order (Majerus, 2013), and theta power increases over these areas is associated with temporal order encoding (Hsieh et al., 2011; Roberts et al., 2013). Thus, fronto-parietal theta oscillations may support memory for temporal order independently of the hippocampus, which is consistent with our findings of preserved temporal order memory in older adults. Separate roles for theta oscillations in distinct networks are consistent with neuroimaging and neuropsychological studies of separate (Amiez \& Petrides, 2007; Kessels et al., 2001; Spiers \& Maguire, 2007) and overlapping (Konkel et al., 2008; Kumaran \& Maguire, 2006) memory traces for spatial and temporal memory.

The present findings speak to the organisation of spatial and temporal memory. A differential effect of ageing was found on memory for spatial versus temporal relations, which if considered alone could be taken as evidence for independent storage of spatial and temporal relations. However, our findings of an effect of temporal information on spatial memory in explicit reports and eye movements, and previous behavioural studies that reported an interaction between spatial and temporal memory (e.g. Delogu et al., 2012a, 2012b; Dutta \& Nairne, 1993), suggest that spatial and temporal relations may not have completely overlapping sources. Indeed, this is consistent with previous neuropsychological (Konkel et al., 2008) and neuroimaging (Kumaran \& Maguire, 2006) studies that provide evidence for a partially overlapping neural trace for spatial and temporal memory. Altogether, this provides converging evidence for a hierarchical organisation of memory. It should be noted that the present evidence for a hierarchical organisation of memory should be interpreted with caution due to the high accuracy of participants in the temporal group. It is possible that if performance of the temporal group was lower, an effect of spatial relations on the explicit reports or eye movements may have been more apparent. Performance for the temporal group remained high even in Experiment 2 despite disruptions to the temporal pacing of the presentation of the objects. 
It may be the case that memory for the temporal relations was consistently more accurate than memory for spatial relations, across both experiments, and for both younger adults, due to how temporal versus spatial relations are represented in memory. In the present study, temporal relations may have been coded verbally (i.e. first, second, and third) in memory, standing in contrast to the likely visuospatial coding of the spatial relations. As a result, the present findings of a one-way dependence of relations in memory, and the implication of a hierarchy in memory, may reflect the hierarchy of verbal versus visual information in memory. Future studies that specifically bias the encoding of both spatial and temporal relations in a visual versus verbal code may determine whether the one-way dependence of relations is observed when the encoding code is held constant. Nonetheless, the findings in the present work point to the intriguing possibility that not all relations may be equal with respect to status in memory, but rather, spatial and temporal relations are organised in a hierarchical structure. The privileged role of temporal relations in memory may be a consequence of how temporal versus spatial information is coded in memory (i.e. verbal versus visual) and/or how the encoding of scenes and episodes unfolds across time.

\section{Acknowledgements}

We would like to thank Doug McQuiggan for assistance in data collection.

\section{Disclosure statement}

No potential conflict of interest was reported by the authors.

\section{Funding information}

This work was supported by the Canadian Institutes of Health Research (CIHR) [grant number MOP126003]; the Natural Sciences and Engineering Research Council of Canada (NSERC) [grant number 482639]. JDR and MDB are supported by the Canada Research Chairs Foundation. MDB is supported by a Scholar Award from the James S McDonnell Foundation. RRII is supported by a graduate fellowship from NSERC.

\section{References}

Amiez, C., \& Petrides, M. (2007).Selective involvement of the mid-dorsolateral prefrontal cortex in the coding of the serial order of visual stimuli in working memory. Proceedings of the National Academy of Sciences of the United States of America, 104(34), 13786-13791. doi:10.1073/pnas.0706220104

Axmacher, N., Cohen, M. X., Fell, J., Haupt, S., Dümpelmann, M., Elger, C. E., ... Ranganath, C. (2010). Intracranial EEG correlates of expectancy and memory formation in the human hippocampus and nucleus accumbens. Neuron, 65(4), 541-549. doi:10.1016/j.neuron.2010.02.006

Axmacher, N., Henseler, M. M., Jensen, O., Weinreich, I., Elger, C. E., \& Fell, J. (2010). Cross-frequency coupling supports multi-item working memory in the human hippocampus. Proceedings of the National Academy of Sciences of the United States of America, 107 (7), 3228-3233.

Blachstein, H., Greenstein, Y., \& Vakil, E. (2012). Aging and temporal order memory: A comparison of direct and indirect measures.
Journal of Clinical and Experimental Neuropsychology, 34(1), 107112. doi:10.1080/13803395.2011.625352

Brandt, S. A., \& Stark, L. W. (1997). Spontaneous eye movements during visual imagery reflect the content of the visual scene. Journal of Cognitive Neuroscience, 9(1), 27-38.

Delogu, F., Nijboer, T. C. W., \& Postma, A. (2012a). Encoding location and serial order in auditory working memory: Evidence for separable processes. Cognitive Processing, 13(3), 267-276. doi:10.1007/ s10339-012-0442-3

Delogu, F., Nijboer, T. C. W., \& Postma, A. (2012b). Binding "when" and "where" impairs temporal, but not spatial recall in auditory and visual working memory. Frontiers in Psychology, 3(March), 62. doi:10.3389/fpsyg.2012.00062

Donaldson, W. (1993). Accuracy of $d^{\prime}$ and $A^{\prime}$ as estimates of sensitivity. Bulletin of the Psychonomic Society, 31(4), 271-274. doi:10.3758/ BF03334926

Driscoll, I., Hamilton, D. A., Petropoulos, H., Yeo, R. A., Brooks, W. M., Baumgartner, R. N., \& Sutherland, R. J. (2003). The aging hippocampus: Cognitive, biochemical and structural findings. Cerebral Cortex, 13(12), 1344-1351. doi:10.1093/cercor/bhg081

Dutta, A., \& Nairne, J. S. (1993). The separability of space and time: Dimensional interaction in the memory trace. Memory \& Cognition, 21(4), 440-448. Retrieved from http://www.ncbi.nlm. nih.gov/pubmed/8350735

Eichenbaum, H. (2014). Time cells in the hippocampus: A new dimension for mapping memories. Nature Reviews Neuroscience, 15(11), 732-744. doi:10.1038/nrn3827

Erickson, K. I., Prakash, R. S., Voss, M. W., Chaddock, L., Heo, S., McLaren, M., ... Kramer, A. F. (2010). Brain-derived neurotrophic factor is associated with age-related decline in hippocampal volume. The Journal of Neuroscience: The Official Journal of the Society for Neuroscience, 30 (15), 5368-5375. doi:10.1523/JNEUROSCI.6251-09.2010

Fell, J., \& Axmacher, N. (2011). The role of phase synchronization in memory processes. Nature Reviews. Neuroscience, 12(2), 105-118. doi:10.1038/nrn2979

Gmeindl, L., Walsh, M., \& Courtney, S. M. (2011). Binding serial order to representations in working memory: A spatial/verbal dissociation. Memory \& Cognition, 39(1), 37-46. doi:10.3758/s13421-010-0012-9

Healy, A. F. (1975). Coding of temporal-spatial patterns in short-term memory. Journal of Verbal Learning and Verbal Behavior, 14(5), 481-495. doi:10.1016/S0022-5371(75)80026-0

Hollingworth, A., Williams, C. C., \& Henderson, J. M. (2001). To see and remember: Visually specific information is retained in memory from previously attended objects in natural scenes. Psychonomic Bulletin \& Review, 8(4), 761-768. doi:10.3758/BF03196215

Howard, M. W., \& Kahana, M. J. (2002). A distributed representation of temporal context. Journal of Mathematical Psychology, 46(3), 269299. doi:10.1006/jmps.2001.1388

Hsieh, L.-T., Ekstrom, A. D., \& Ranganath, C. (2011). Neural oscillations associated with item and temporal order maintenance in working memory. The Journal of Neuroscience: The Official Journal of the Society for Neuroscience, 31(30), 10803-10810. doi:10.1523/ JNEUROSCI.0828-11.2011

Jensen, O., \& Colgin, L. L. (2007). Cross-frequency coupling between neuronal oscillations. Trends in Cognitive Sciences, 11(7), 267-269. doi:10.1016/j.tics.2007.05.003

Kahana, M. J., Howard, M. W., \& Polyn, S. M. (2008). Associative retrieval processes in episodic memory. In H. L. Roediger III (Ed.), Cognitive psychology of memory. Vol. 2 of Learning and memory: A comprehensive reference (J. Byrne, Editor) (pp. 476-490). Oxford: Elsevier.

Kahana, M. J., Howard, M. W., Zaromb, F., \& Wingfield, A. (2002). Age dissociates recency and lag recency effects in free recall. Journal of Experimental Psychology: Learning, Memory, and Cognition, 28 (3), 530-540. doi:10.1037//0278-7393.28.3.530

Kahana, M. J., Sekuler, R., Caplan, J. B., Kirschen, M., \& Madsen, J. R. (1999). Human theta oscillations exhibit task dependence during virtual maze navigation used (positron emission tomography and functional magnetic. Nature, 399(June), 781-784. 
Kessels, R. P. C., de Haan, E. H. F., Kappelle, L. J., \& Postma, A. (2001). Varieties of human spatial memory: A meta-analysis on the effects of hippocampal lesions. Brain Research Reviews, 35(3), 295303. doi:10.1016/S0165-0173(01)00058-3

Konkel, A., Warren, D. E., Duff, M. C., Tranel, D. N., \& Cohen, N. J. (2008). Hippocampal amnesia impairs all manner of relational memory. Frontiers in Human Neuroscience, 2(October), 15. doi:10.3389/ neuro.09.015.2008

Kopelman, M. D., Stanhope, N., \& Kingsley, D. (1997). Temporal and spatial context memory in patients with focal frontal, temporal lobe, and diencephalic lesions. Neuropsychologia, 35(12), 15331545. doi:10.1016/S0028-3932(97)00167-X

Kumaran, D., \& Maguire, E. a. (2006). An unexpected sequence of events: Mismatch detection in the human hippocampus. PLoS Biology, 4(12), e424. doi:10.1371/journal.pbio.0040424

Laeng, B., \& Teodorescu, D.-S. (2002). Eye scanpath during visual imagery reenact those of perception of the same visual scene. Cognitive Science, 26, 207-231.

Lega, B. C., Jacobs, J., \& Kahana, M. (2012). Human hippocampal theta oscillations and the formation of episodic memories. Hippocampus, 22(4), 748-761. doi:10.1002/hipo.20937

Lisman, J., \& Buzsáki, G. (2008). A neural coding scheme formed by the combined function of gamma and theta oscillations. Schizophrenia Bulletin, 34(5), 974-980. doi:10.1093/schbul/sbn060

Lisman, J. E., \& Idiart, M. A. P. (1995). Store of 7 +/- 2 short-term memories in oscillatory subcycles. Science, 267(5203), 1512-1515.

Lisman, J. E., \& Redish, A. D. (2011). Prediction, sequences, and the hippocampus. In M. Bar (Ed.), Predictions in the brain: Using our past to generate a future (pp. 258-270). New York, NY: Oxford University Press.

Majerus, S. (2013). Language repetition and short-term memory: An integrative framework. Frontiers in Human Neuroscience, 7(July), 116. doi:10.3389/fnhum.2013.00357

Mueller, S. T., \& Weidemann, C. T. (2008). Decision noise: An explanation for observed violations of signal detection theory. Psychonomic Bulletin \& Review, 15(3), 465-494. doi:10.3758/PBR.15.3.465

Mueller, S. T., \& Zhang, J. (2005). Upper and lower bounds of area under ROC curves and index of discriminability of classifier performance. In Proc. of the ICML 2006 workshop on ROC analysis in Machine Learning, 41-46. Retrieved from http://users.dsic.upv.es/ flip/ ROCML2006/Papers/Proc_ROCML2006.pdf

O'Keefe, J., \& Nadel, L. (1978) The hippocampus as a cognitive map. Oxford: Clarendon Press.

O'Keefe, J., \& Recce, M. L. (1993). Phase relationship between hippocampal place units and the EEG theta rhythm. Hippocampus, 3(3), 317-330. doi:10.1002/hipo.450030307

Old, S. R., \& Naveh-Benjamin, M. (2008). Differential effects of age on item and associative measures of memory: A meta-analysis. Psychology and Aging, 23(1), 104-118. doi:10.1037/0882-7974.23.1.104

Olsen, R. K., Moses, S. N., Riggs, L., \& Ryan, J. D. (2012). The hippocampus supports multiple cognitive processes through relational binding and comparison. Frontiers in Human Neuroscience, 6(May), 1-13. doi:10.3389/fnhum.2012.00146

Olsen, R. K., Rondina II R., Riggs, L., Meltzer, J. A., \& Ryan, J. D. (2013). Hippocampal and neocortical oscillatory contributions to visuospatial binding and comparison. Journal of Experimental Psychology. General, 142(4), 1335-1345. doi:10.1037/a0034043

Olson, I. R., Zhang, J. X., Mitchell, K. J., Johnson, M. K., Bloise, S. M., \& Higgins, J. A. (2004). Preserved spatial memory over brief intervals in older adults. Psychology and Aging, 19(2), 310-317. doi:10.1037/ 0882-7974.19.2.310

Parker, R. E. (1978). Picture processing during recognition. Journal of Experimental Psychology. Human Perception and Performance, 4(2), 284-293. doi:10.1037/0096-1523.4.2.284

Parkin, A. J., Walter, B. M., \& Hunkin, N. M. (1995). Relationships between normal aging, frontal lobe function, and memory for temporal and spatial information. Neuropsychology, 9(3), 304-312. doi:10.1037/0894-4105.9.3.304
Pathman, T., \& Ghetti, S. (2014). The eyes know time: A novel paradigm to reveal the development of temporal memory. Child Development, 85(2), 792-807. doi:10.1111/cdev.12152

Pathman, T., \& Ghetti, S. (2015). Eye movements provide an index of veridical memory for temporal order. Plos One, 10(5), e0125648. doi:10.1371/journal.pone. 0125648

Raghavachari, S., Kahana, M. J., Rizzuto, D. S., Caplan, J. B., Kirschen, M. P., Bourgeois, B., ... Lisman, J. E. (2001). Gating of human theta oscillations by a working memory task. The Journal of Neuroscience: The Official Journal of the Society for Neuroscience, 21(9), 3175-3183. Retrieved from http://www.ncbi.nlm.nih.gov/pubmed/11312302

Roberts, B. M., Hsieh, L.-T., \& Ranganath, C. (2013). Oscillatory activity during maintenance of spatial and temporal information in working memory. Neuropsychologia, 51(2), 349-357. doi:10.1016/j. neuropsychologia.2012.10.009

Rondina II, R., Olsen, R. K., McQuiggan, D. A., Fatima, Z., Li, L., Oziel, E., ... Ryan, J. D. (2015) Age-related changes to oscillatory dynamics in hippocampal and neocortical networks. Neurobiology of Learning and Memory. doi:10.1016/j.nlm.2015.11.017

Ryan, J. D., Althoff, R. R., Whitlow, S., \& Cohen, N. J. (2000). Amnesia is a deficit in relational memory. Psychological Science, 11(6), 454-461. Retrieved from http://www.ncbi.nlm.nih.gov/pubmed/11202489

Ryan, J. D., \& Cohen, N. J. (2004). The nature of change detection and online representations of scenes. Journal of Experimental Psychology. Human Perception and Performance, 30(5), 988-1015. doi:10.1037/0096-1523.30.5.988

Ryan, J. D., Hannula, D. E., \& Cohen, N. J. (2007). The obligatory effects of memory on eye movements. Memory (Hove, England), 15(5), 508525. doi:10.1080/09658210701391022

Ryan, J. D., Leung, G., Turk-Browne, N. B., \& Hasher, L. (2007). Assessment of age-related changes in inhibition and binding using eye movement monitoring. Psychology and Aging, 22(2), 239-250. doi:10.1037/0882-7974.22.2.239

Ryan, J. D., \& Villate, C. (2009). Building visual representations: The binding of relative spatial relations across time. Visual Cognition, 17(1-2), 254-272. doi:10.1080/13506280802336362

Sauseng, P., Griesmayr, B., Freunberger, R., \& Klimesch, W. (2010). Control mechanisms in working memory: A possible function of EEG theta oscillations. Neuroscience and Biobehavioral Reviews, 34 (7), 1015-1022. doi:10.1016/j.neubiorev.2009.12.006

Sederberg, P. B., Miller, J. F., Howard, M. W., \& Kahana, M. J. (2010). The temporal contiguity effect predicts episodic memory performance. Memory \& Cognition, 38(6), 689-699. doi:10.3758/MC.38.6.689

Sekuler, R., McLaughlin, C., Kahana, M. J., Wingfield, A., \& Yotsumoto, Y. (2006). Short-term visual recognition and temporal order memory are both well-preserved in aging. Psychology and Aging, 21(3), 632-637. doi:10.1037/0882-7974.21.3.632

Spiers, H. J., \& Maguire, E. a. (2007). Decoding human brain activity during real-world experiences. Trends in Cognitive Sciences, 11(8), 356-365. doi:10.1016/j.tics.2007.06.002

Stanislaw, H., \& Todorov, N. (1999). Calculation of signal detection theory measures. Behavior Research Methods, Instruments, \& Computers, 31(1), 137-149. doi:10.3758/BF03207704

Van Asselen, M., Van der Lubbe, R. H. J., \& Postma, A. (2006). Are space and time automatically integrated in episodic memory? Memory (Hove, England), 14(2), 232-240. doi:10.1080/09658210500172839

Walker-Smith, G. J., Gale, A. G., \& Findlay, J. M. (1977). Eye movement strategies involved in face perception. Preception, 6(3), 313-326.

Weidemann, C. T., \& Mueller, S. T. (2008). Decision noise may mask criterion shifts: Reply to Balakrishnan and MacDonald (2008). Psychonomic Bulletin \& Review, 15(5), 1031-1034. doi:10.3758/PBR. 15.5.1031

Yarbus, A. L. (1967). Eye movements and vision. New York, NY: Plenum Press.

Zhang, J., \& Mueller, S. T. (2005). A note on ROC analysis and non-parametric estimate of sensitivity. Psychometrika, 70(1), 145-154. doi:10. 1007/s11336-003-1119-8 